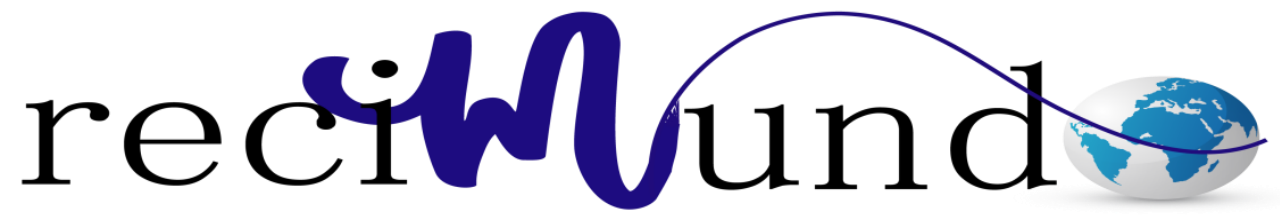

Revista Cientifica Mundo de la Investigación y el Conocimiento

Edgar Fredy Morales Caguana ${ }^{\text {a }}$ Juan Carlos Guevara Espinoza ${ }^{\text {b }}$ Claudio Federico Malo Toledo ${ }^{\mathrm{c}}$

Lectura rápida: Herramienta fundamental en la Educación Superior

Revista Científica Mundo de la Investigación y el Conocimiento. Vol. 2 núm., especial, mayo, ISSN: 2588-073X, 2018, pp. 300-317

DOI: 10.26820/recimundo/2.esp.2018.300-317

Editorial Saberes del Conocimiento

Recibido: 05/12/2017 Aceptado: $15 / 02 / 2018$

a. Universidad de Guayaquil; edgar.moralesc@ug.edu.ec

b. Universidad de Guayaquil; juan.guevara@ug.edu.ec

c. Universidad de Guayaquil; cludio.malot@ug.edu.ec 


\section{Lectura rápida: Herramienta fundamental en la Educación Superior}

Vol. 2, núm. Esp., (2018)

Edgar Fredy Morales Caguana; Juan Carlos Guevara Espinoza; Claudio Federico Malo Toledo

\section{RESUMEN}

La presente investigación se realizó con la finalidad de explicar de qué se trata la lectura rápida y a su vez de proporcionar el conocimiento básico que sirva de referencia para que el estudiante de educación superior comprenda sus tipos e importancia, animándolo a desarrollar dicha herramienta de aprendizaje, considerada como una habilidad fundamental a nivel de estudios superiores ya que se entiende que su dominio posibilita un mejor acceso y comprensión de información. En buena medida los conocimientos que adquiere un estudiante, los capta a través de la lectura, durante el proceso de enseñanza-aprendizaje, se necesita leer una variada gama de textos para apropiarse de diferentes conocimientos y la importancia del hecho, no sólo radica en el contenido, sino en la cantidad, el estilo y hasta los propósitos de cada lectura. La metodología empleada en la presente investigación fue con base en un enfoque documental, a nivel exploratorio-descriptivo, mediante una revisión bibliográfica laboriosa que incluyó la búsqueda tanto en publicaciones hechas en material físico como electrónicos, tales como libros, revistas especializadas, foros, simposios, páginas web, e-book y demás fuentes de información, nacionales e internacionales, legales, oficiales y extraoficiales, respaldadas por profesionales en la materia, en idioma castellano, y en todo momento, relacionadas con el tema en cuestión, todo ello con la primordial intención de que la información obtenida sirva para proporcionar conocimiento fundamental al interesado, refiriendo y desarrollando conceptos tales como: y particularmente en esta investigación, para que sirva de fundamento en valiosas conclusiones que se desarrollarán a continuación.

Palabras Clave: Lectura, aprendizaje, habilidad, comprensión, conocimientos. 


\title{
Lectura rápida: Herramienta fundamental en la Educación Superior
}

Vol. 2, núm. Esp., (2018)

Edgar Fredy Morales Caguana; Juan Carlos Guevara Espinoza; Claudio Federico Malo Toledo

\begin{abstract}
The present investigation was carried out with the purpose of explaining what fast reading is about and at the same time to provide the basic knowledge that serves as reference so that the student of higher education understands their types and importance, encouraging him to develop this learning tool, considered as a fundamental skill at the level of higher education since it is understood that its domain enables better access and understanding of information. To a large extent, the knowledge that a student acquires, captures through reading, during the teachinglearning process, you need to read a wide range of texts to appropriate different knowledge and the importance of the fact, not only lies in the content, but in the quantity, style and even the purposes of each reading. The methodology used in the present investigation was based on a documentary approach, at the exploratory-descriptive level, through a laborious bibliographic review that included the search both in publications made in physical and electronic material, such as books, specialized magazines, forums, symposiums, web pages, e-book and other sources of information, national and international, legal, official and unofficial, supported by professionals in the field, in Spanish, and at all times, related to the subject in question, all with the Primordial intention that the information obtained serve to provide fundamental knowledge to the interested party, referring and developing concepts such as: and particularly in this investigation, to serve as a basis for valuable conclusions that will be developed below.
\end{abstract}

Keywords: Reading, learning, skill, understanding, knowledge. 


\section{Lectura rápida: Herramienta fundamental en la Educación Superior}

Vol. 2, núm. Esp., (2018)

Edgar Fredy Morales Caguana; Juan Carlos Guevara Espinoza; Claudio Federico Malo Toledo

\section{Introducción.}

Gran parte de la enseñanza en escuelas y universidades se realiza a través de la comunicación escrita en libros, artículos, apuntes. Por esta razón es de suma importancia que los estudiantes aprendan a manejar muy bien estos documentos para sacarles el máximo provecho con la lectura de los mismos.

Mayormente, los conocimientos que adquiere un estudiante, los capta a través de la lectura, durante el proceso de enseñanza-aprendizaje, desde la primaria hasta la educación superior, debido a que se necesita leer una variada gama de textos para apropiarse de diferentes conocimientos, por lo que la importancia del hecho, radica no sólo en el contenido, sino también en la cantidad, el estilo y hasta los propósitos de la lectura.

El buen uso de las habilidades para la lectura comprensiva y el manejo de textos técnicos y científicos, sin duda, aseguran un éxito y progreso académico para los estudiantes. Así mismo, el conocimiento de las técnicas de comprensión de lectura, asegura a los profesionales de hoy en día ser competitivos en su vida laboral.

No solamente en el ámbito de la vida profesional o en la escuela la comunicación escrita es importante, sino también en la vida cotidiana. Las personas estamos en constante aprendizaje de las cosas que suceden a nuestro alrededor y para ello necesitamos leer diarios y revistas, por lo que también se necesitan buenas habilidades de lectura comprensiva. O simplemente para el ocio en el tiempo libre, etc.

Leer no implica solamente enterarse del contenido de un texto como lo hacen estudiantes de primeria y más firmemente un estudiante de secundaria que solo logran el desciframiento del 


\section{Lectura rápida: Herramienta fundamental en la Educación Superior}

Vol. 2, núm. Esp., (2018)

Edgar Fredy Morales Caguana; Juan Carlos Guevara Espinoza; Claudio Federico Malo Toledo

texto. Esto solo sirve para leer anuncios, propagandas, etc. Para una persona de educación superior, leer implica interactuar con la información, dar un valor a lo aprendido, razonar con la lectura.

Aprender a leer más rápido puede ser beneficio tanto para la formación universitaria como para el desarrollo laboral, dado que permite mejorar la comprensión lectora y liberar tiempo para trabajar de forma más eficiente en otras áreas. Comprender un libro de texto no significa que seamos buenos lectores. Ser un buen lector implica leer rápido, con eficiencia y entendimiento.

Dicho esto, es que se puede entrar en la materia que este caso nos ocupa, la lectura rápida. Lógicamente para comprenderla es necesario desarrollar previamente algunos conceptos importantes, tales como: lectura, comprensión lectora, rasgos del buen lector, lectura rápida, conceptualizaciones generales, Adquisición de mayor ritmo lector y consejos.

\section{Materiales y métodos}

\section{Materiales}

Materiales de escritorio, material bibliográfico, materiales audiovisuales, materiales para impresión.

\section{Métodos}

En vista de que el objetivo de este trabajo investigativo es describir, definir términos y conceptualizar en líneas generales respecto a la lectura rápida, haciendo énfasis en la importancia 


\section{Lectura rápida: Herramienta fundamental en la Educación Superior}

Vol. 2, núm. Esp., (2018)

Edgar Fredy Morales Caguana; Juan Carlos Guevara Espinoza; Claudio Federico Malo Toledo

de ésta como herramienta fundamental en la educación superior, se ha propuesto el desarrollo de una investigación de tipo documental, a nivel exploratoria, de corte longitudinal.

Respecto a la investigación documental, Baena (1985) citado en Ávila (2006), la define como "una técnica que consiste en la selección y recopilación de información por medio de la lectura y crítica de documentos y materiales bibliográficos, de bibliotecas, hemerotecas, centros de documentación e información” (p. 72).

Franklin (1997), igualmente citado en Ávila (2006), respecto a la investigación documental aplicada a la organización de empresas como una técnica de investigación, dice que "se deben seleccionar y analizar aquellos escritos que contienen datos de interés relacionados con el estudio" (p. 13).

Según Ávila (2006) en los Estudios Longitudinales la unidad de análisis es observada en varios puntos en el tiempo.

Parafraseando a Ríos (2017) se entiende que la investigación se cataloga como documental porque responde a una fuente de información, puesto que se apela a documentos para alcanzar los resultados planeados; y en cuanto al nivel de conocimiento, la investigación es descriptiva, cuando busca encontrar las características, comportamiento y propiedades del objeto de estudio en el presente, no ha futuro, siendo en ese último caso que se denomina investigación de pronóstico. 


\section{Lectura rápida: Herramienta fundamental en la Educación Superior}

Vol. 2, núm. Esp., (2018)

Edgar Fredy Morales Caguana; Juan Carlos Guevara Espinoza; Claudio Federico Malo Toledo

\section{Resultados.}

\section{La lectura}

Según García (2008), la lectura está íntimamente relacionada con la educación, y es en la escuela donde verdaderamente se forma el lector, ocurre con frecuencia que el hábito de la lectura se olvida a medida que los estudiantes van promocionando, por lo que muchos de ellos no desarrollan una buena comprensión lectora. Desde este punto de vista, la lectura requiere de un proceso de aprendizaje, y como tal, se logra a través de un proceso gradual en el que se va adquiriendo. Cabe destacar que, no es inherente al cerebro humano, sino que debe ser aprendida y autorizada.

Sin embargo, señala López (2008) pasa igual que el lector normal por las mismas etapas de lectura, con la diferencia que se queda varado en la fase alfabética y sólo se logra desarrollar las posteriores en forma imperfecta. Así pues, con tratamiento instruccional podrá avanzar en forma eficiente por cada una y cuanto más temprano sea la intervención, mayores son las probabilidades de lograr una mejor automatización y menores las probabilidades de que adquiera vicios de lectura.

\section{Comprensión Lectora}

Definir la comprensión lectora es un término que ha ido evolucionando con el tiempo y las investigaciones. La comprensión lectora lejos de ser una tarea sencilla, se considera un proceso complejo, que pone en juego diversos factores y habilidades, como el reconocimiento de palabras y a la comprensión del lenguaje, debiendo se aplicar automáticamente y simultáneamente para darle una lectura experta. 


\section{Lectura rápida: Herramienta fundamental en la Educación Superior}

Vol. 2, núm. Esp., (2018)

Edgar Fredy Morales Caguana; Juan Carlos Guevara Espinoza; Claudio Federico Malo Toledo

Hernández y Quintero (2011), señalan que una manera de entender la comprensión de un texto es a través de los siguientes aspectos importantes: En primer lugar, romper con interpretaciones simplistas propugnadas anteriormente desde los modelos lineales. Comprender no consiste solo en extraer el significado del material escrito, ya que tal definición solo otorgaría relevancia a uno de los factores que interviene en el proceso del texto, considerándose entonces que son las características y naturaleza específicas de éste las que están condicionando la comprensión. Comprender, desde la perspectiva interactiva, implica la reconstrucción personal de significado: construcción guiada tanto por los conocimientos previos, los objetivos, el contexto, entre otros; como por las estrategias que pone en juego el lector durante todo el proceso.

En segundo lugar, caracterizar a la comprensión como un proceso constructivo personal, la interpretación que el sujeto haga de la información contenida en el texto, no será nunca única y estable, como se defiende desde los modelos lineales, sino que se presta a distintos significados potenciales dependiendo de las contribuciones y situaciones del lector, quien construirá activamente algunas de las representaciones mentales posibles.

En tercer lugar, hacer una interpretación interactiva de la comprensión, obligando a adoptar posiciones y decisiones didácticas muy diferentes a las asumidas desde los modelos lineales. Se impone una instrucción que enfatice el carácter constructivo por parte del lector. Dicha instrucción es imprescindible si se admite que manejar hábilmente el código propio del lenguaje escrito es una condición necesaria, pero no suficiente, para que se produzca la atribución personal de significados. 


\section{Lectura rápida: Herramienta fundamental en la Educación Superior}

Vol. 2, núm. Esp., (2018)

Edgar Fredy Morales Caguana; Juan Carlos Guevara Espinoza; Claudio Federico Malo Toledo

\section{Lectura Rápida}

Indica Ruiz (2008) que, para obtener una comprensión sólida del contenido de un texto en poco tiempo, importan más las ideas principales que los detalles específicos. Es importante hacer notar que, existen dos tipos de lectura rápida: lectura global: que se define como una técnica que se usa cuando el lector quiere extraer la idea o ideas más importantes de un texto; y la lectura selectiva: que se usa cuando el lector quiere localizar una información concreta sin necesidad de entender el resto del texto o pasaje, es decir, se usa para encontrar una información en concreto.

Una lectura rápida, según Beas (2006), no solo se consigue con un movimiento adecuado de los ojos, se debe: ampliar el vocabulario y con ello incrementar capacidad de la comprensión de lo leído y hacer una lectura inteligente. La lectura rápida solo es posible cuando estamos muy familiarizados con el lenguaje y su contenido.

\section{Conceptualización General}

Luego de consultar la obra de Guevara, Bilbao, Cárdenas, \& Delgado (2011) se puede comprender que, al analizar los distintos tipos de lectura, se aprecian diferencias en la clasificaciones ofrecidas por los distintos autores consultados, sin embargo, también se puede decir, que todos coinciden en que dichas clasificaciones dependen de los objetivos que persiga el lector.

Teniendo claro esto, se puede estar dispuesto a mencionar aquellas que se consideran de mayor relevancia para el presente caso, por ejemplo, la clasificación propuesta por Antich (1988) citado por los autores antes mencionados, describe varios tipos de lectura en general, según diversos criterios, que son: 


\section{Lectura rápida: Herramienta fundamental en la Educación Superior}

Vol. 2, núm. Esp., (2018)

Edgar Fredy Morales Caguana; Juan Carlos Guevara Espinoza; Claudio Federico Malo Toledo

\section{Según el objetivo o propósito del lector, la lectura puede ser:}

a. De información general: es la que se realiza al leer un texto que se desconoce total o parcialmente, y sobre lo cual se desea obtener conocimientos. Ejemplos: la lectura de noticias del periódico, informaciones científicos o técnicos de tipo divulgatorio, artículo de divulgación general, hechos históricos. Se hace en textos de carácter informativo general, de temas no muy conocidos por el estudiantado. En el trabajo de investigación científica esta lectura se llama también de revisión.

b. De estudio u observación: tiene lugar cuando se lee detenidamente para aprender y retener los materiales y se caracteriza por ser bastante lenta. Siempre debe mantenerse una primera lectura inicial rápida y silenciosa para captar el todo antes que las partes, requiere prácticamente el ciento por ciento de compresión, ejemplo la lectura de libros de textos, de algunos materiales de referencia que se consultan para estudios.

c. De búsqueda de información específica: se realiza cuando el tema es conocido en general y el lector desea encontrar lo que proporciona de nuevo, es lo contrario de la lectura de información general. Requiere textos que contengan otros contextos, tales como nombres, fechas, cantidades, acuerdos y conocimientos previos del tema, lo cual se verifica mediante una o dos preguntas previas generales.

d. De recreación: se realiza con materiales anecdóticos, de contenido humorístico, de ciencia ficción, crónicas de viajes, aventuras, relatos históricos, poemas.

\section{Según el proceso mental la lectura puede ser:}




\section{Lectura rápida: Herramienta fundamental en la Educación Superior}

Vol. 2, núm. Esp., (2018)

Edgar Fredy Morales Caguana; Juan Carlos Guevara Espinoza; Claudio Federico Malo Toledo

e. Sintética: puede ser silenciosa u oral. La lectura oral debe proceder a la silenciosa debido a que permite dominar el sistema de sonidos de la lengua y por ende, desarrollar la lectura.

f. Analítica: se utiliza primeramente para explotar los contenidos lingüísticos tanto fonológicos como léxicos gramaticales; y cumple una función instrumental. En un segundo momento se inicia el trabajo para el desarrollo del mecanismo de inferencia de significado de las palabras, contribuyendo al desarrollo y al enriquecimiento del léxico.

3. Según la organización pedagógica:

g. Lectura en clase: esta lectura constituye un aspecto fundamental de los cursos de idiomas.

h. Lectura extractase: su objetivo es reforzar el desarrollo de las habilidades, estimular la independencia cognoscitiva de lectura y crear hábitos de trabajo independiente.

4. Según el modo de realización por el alumno:

i. Lectura oral: tiene como fin reforzar el conocimiento del sistema de correspondencia grafía - sonido, ejercitar la pronunciación y aprender a leer por grupos de palabras, y se realiza en el nivel elemental del aprendizaje. Esta lectura que realiza el alumno le sirve al profesor en cierta medida para comprobar la comprensión.

j. Lectura silenciosa: tiene como fin obtener información. Es la que refleja el proceso normal de lectura y se realiza en clase o fuera de ella después de pasado el nivel elemental. 


\section{Lectura rápida: Herramienta fundamental en la Educación Superior}

Vol. 2, núm. Esp., (2018)

Edgar Fredy Morales Caguana; Juan Carlos Guevara Espinoza; Claudio Federico Malo Toledo

Cassany, Luna, \& Sanz (1998), definen algunos rasgos del buen lector o lector competente en los siguientes términos:

$\checkmark$ Lee habitualmente en silencio, pero también puede oralizar, si es necesario.

$\checkmark$ No cae en los defectos típicos de lectura: oralización, subvocalización, regresiones, etc.

$\checkmark$ Lee con rapidez y eficientemente. Hace fijaciones amplias, rápidas y selectivas.

$\checkmark$ Se fija en unidades superiores del texto: palabras, frases, etc.

$\checkmark$ No repasa el texto letra por letra.

Adquisición de mayor ritmo lector

Iglesias (2009) argumenta que la velocidad lectora, se trata de destrezas físicas y mentales que necesitan método y práctica.

Andrade (2003) sostiene que el lector que ansíe progresar en ganar velocidad debe hacerlo en forma sistemática y metódica; paso a paso y de un nivel a otro por vez. A la par que la velocidad, debe impulsarse el desarrollo de técnicas adecuadas de comprensión, concentración y retención. Insiste en la necesidad de practicar. Sólo la práctica hace dueño del secreto de la lectura veloz.

Consejos

De conformidad a la literatura consultada, se exponen a continuación algunos consejos para aumentar la capacidad de lectura y para deshacerse de los malos hábitos de lectura más comunes. (Universia Argentina, 2016) 


\section{Lectura rápida: Herramienta fundamental en la Educación Superior}

Vol. 2, núm. Esp., (2018)

Edgar Fredy Morales Caguana; Juan Carlos Guevara Espinoza; Claudio Federico Malo Toledo

\section{Sub-vocalización:}

Es la costumbre que tiene la mayoría de la gente de pronunciar las palabras en la cabeza mientras lee el texto. Cuando uno escucha la palabra que dice la mente, la lectura toma mucho más tiempo de la necesaria porque una palabra se puede entender más rápidamente de lo que se puede decir. Por lo tanto, hay que terminar con el hábito de la sub-vocalización y practicar "no hablar" o dejar de decir las palabras en la cabeza cuando se lee Lectura de palabra por palabra:

Es más lento leer palabra por palabra. Cuando uno se concentra en las palabras por separado, muchas veces estas pierden el contexto general y en definitiva el concepto de lo que se dice. Por lo tanto, la gente que lee cada palabra como una unidad distinta tiene menos comprensión lectora que quienes hacen una lectura en bloques de palabras. Cuantas más palabras se puedan leer en cada bloque, más rápida será la lectura.

\section{Regresión}

La regresión es la re-lectura innecesaria de material. Algunas personas tienen el hábito de volver a leer lo que acaban de leer solamente para asegurarse de estar haciéndolo bien. A pesar de que así muchas veces pensamos que comprenderemos mejor el texto, solo perdemos la estructura general y el flujo de lectura con el que veníamos y al final, la comprensión lectora puede disminuir. Hay que ser conscientes del uso de la regresión y no volver a leer el material a no ser que sea absolutamente necesario. 


\section{Lectura rápida: Herramienta fundamental en la Educación Superior}

Vol. 2, núm. Esp., (2018)

Edgar Fredy Morales Caguana; Juan Carlos Guevara Espinoza; Claudio Federico Malo Toledo

\section{Pobre concentración}

Para mejorar la concentración al leer, es necesario dejar de realizar múltiples tareas y eliminar todo tipo de distracciones. La lectura debe realizarse en un ambiente adecuado, donde las distracciones externas sean mínimas.

\section{Lectura lineal}

Siempre se nos ha enseñado a leer comenzando por la izquierda y finalizando por la derecha y siempre hacia abajo, cada palabra, oración, párrafo y página en secuencia. Sin embargo, no hay una regla que diga cómo tenemos que leer un documento o que hay que hacerlo en el orden que el autor pretende. Uno puede escanear rápidamente el texto y así decidir lo que es importante y lo que no lo es.

Algunos consejos adicionales para mejorar la comprensión de la lectura y la velocidad, según Zorrilla (2008) son:

$\checkmark$ Intentar mejorar el vocabulario, consultar frecuentemente el diccionario.

$\checkmark$ Hacer fichas de las nuevas palabras aprendidas y utilizarlas.

No leer más rápido de lo que permita la comprensión. La velocidad de la lectura no es un reto en sí mismo. Lo es, sin embargo, la comprensión.

$\checkmark$ Practicar la lectura durante tres o cuatro semanas intentando mejorar la comprensión y rapidez durante 15 minutos cada día.

$\checkmark$ Cada semana, cronometrar la lectura de un capítulo de un libro y medir el número de páginas por hora leído. 


\section{Lectura rápida: Herramienta fundamental en la Educación Superior}

Vol. 2, núm. Esp., (2018)

Edgar Fredy Morales Caguana; Juan Carlos Guevara Espinoza; Claudio Federico Malo Toledo

La velocidad en la lectura normal depende de los fines y su unidad de medida se expresa en palabras por minuto (ppm):

- Para memorización, menos de 100 ppm

- De aprendizaje (100-200 ppm)

- De comprensión (200-400ppm)

- Informativa (400-700 ppm)

Importante es tener claro que, entre los diversos tipos de lectura, la de comprensión es probablemente el proceso más importante, ya que es la que motiva la lectura cotidiana de la mayor parte de la gente. En cambio, la lectura veloz es útil para procesar superficialmente grandes cantidades de texto, pero está por debajo del nivel de comprensión.

\section{Técnicas de la lectura}

Romero et al. (2016) dice que, generalmente, se necesita que los estudiantes lean textos muy extensos y complicados de entender para la enseñanza de la lectura. Igualmente, cita a Beas (2006) quien al respecto refiriere que "se trata de lecturas obligatorias para el conocimiento previo de cada una de las asignaturas. Para verificar el adecuado nivel de comprensión, se puede utilizar diversas técnicas, antes de aplicar las técnicas de lectura, se debe enseñar a los alumnos para qué sirven sus estrategias de elaboración y ejemplos sencillos para que cada estudiante, diseñe individualmente, la técnica de comprensión lectora que va a utilizar.

Seguidamente en la misma literatura se hace referencia a Alcega (2008), quien asegura que el defecto más generalizado al que se enfrentan los lectores más jóvenes es un excesivo análisis de las palabras o la lectura palabra por palabra. Agrega la misma autora que a veces esta 


\section{Lectura rápida: Herramienta fundamental en la Educación Superior}

Vol. 2, núm. Esp., (2018)

Edgar Fredy Morales Caguana; Juan Carlos Guevara Espinoza; Claudio Federico Malo Toledo

costumbre se acompaña por movimientos de labios y de boca, lo que hay que corregir a toda costa si se quiere mejorar la velocidad de lectura. Para la autora, tanto la velocidad como la comprensión pueden mejorarse ampliamente con el ejercicio. En el mismo orden de ideas, es López (2008) el citado por (Romero, Barboza, Cueva, Arenas, \& Cuevas, 2016), debido a que éste señala que para favorecer la comprensión lectora como la velocidad, hay que aplicar las siguientes técnicas:

- Buscar las ideas y su encadenamiento lógico.

- No pararse en las palabras sino buscar el mensaje que encierran.

- No vocalizar al leer, pues eso dificulta la captación de las ideas.

- Captar el sentido del texto, no leer todas las palabras.

- Consultar a menudo el diccionario.

- Anotar en un cuaderno las palabras y expresiones nuevas que se encuentren.

- Leer gráficos, esquemas e ilustraciones, facilita la comprensión de los textos.

- Los gráficos o esquemas pueden resultar claros e ilustrativos que muchas proposiciones escritas, cuyo mensaje semántico es más complejo.

Así pues, estas técnicas ayudarán a tener una mejor comprensión lectora a través de mapas conceptuales y a realizar una lectura rápida del texto. Según los autores tratados anteriormente, hay distintas técnicas de lectura que sirven para adaptar la manera de leer al objetivo que persigue el lector. Las dos intenciones más comunes al leer son la maximización de la velocidad y la maximización de comprensión del texto. En general estos objetivos son contrarios y es necesario concertar un balance entre los dos. 


\section{Lectura rápida: Herramienta fundamental en la Educación Superior}

Vol. 2, núm. Esp., (2018)

Edgar Fredy Morales Caguana; Juan Carlos Guevara Espinoza; Claudio Federico Malo Toledo

\section{Conclusiones.}

La lectura veloz es útil para procesar superficialmente grandes cantidades de texto.

Tanto la velocidad de lectura como la comprensión pueden mejorarse ampliamente con el ejercicio.

Lleva tiempo optimizar las habilidades de lectura, por ello, es aconsejable que antes de comenzar a practicar, se mida la velocidad de lectura actual para así saber si ésta aumenta.

Si es necesario comprender el mensaje completo, memorizar la información, analizar en detalle o simplemente disfrutar de la forma en que escribe un autor; la lectura veloz no es adecuada.

La velocidad de lectura puede mejorar significativamente la eficacia y comprensión global del texto, cuando se aplica correctamente.

Si se quiere mejorar la velocidad de lectura, hay que corregir a toda costa el movimiento de labios y de boca.

Siempre hay que tener en cuenta el propósito de la lectura y decidir si la lectura veloz es el enfoque más adecuado ya que no todo lo que uno lee se presta para una lectura rápida.

\section{Bibliografía.}

Alcega, S. (2008). Técnicas y estrategias para mejorar la comprensión lectora. Pedagogía Terapéutica. Caracas - Venezuela.

Andrade. (2003). Lectura y aprendizaje eficaz. Lima: Andrade S.R.L.

Ávila, H. (2006). Introducción a la Metodología de la Investigación. eumed.net. 


\section{Lectura rápida: Herramienta fundamental en la Educación Superior}

Vol. 2, núm. Esp., (2018)

Edgar Fredy Morales Caguana; Juan Carlos Guevara Espinoza; Claudio Federico Malo Toledo

Beas, J. (2006). Enseñar a pensar para aprender mejor. Santiafo de Chile - Chile: Universidad Católica de Chile.

Cassany, D., Luna, M., \& Sanz, G. (1998). Enseñar Lengua. Madrid: Grao.

García, J. (2008). Comprensión y mejora de textos. Madrid - España: Ediciones Visor.

Guevara, G., Bilbao, B., Cárdenas, B., \& Delgado, M. (2011). Hacia Una Lectura Superior: La Habilidad De Leer. (J. C. Coll, Ed.) Contribuciones a las Ciencias Sociales. Recuperado el 30 de 03 de 2018, de http://www.eumed.net/rev/cccss/16/gbbm.html

Hernández, A., \& Quintero, A. (2011). Estrategias para fortalecer la comprensión y el hábito de la lectura. Lima - Perú: Bruño.

Iglesias, J. (2009). Ritmo ideal de la lectura silenciosa. Pueblo cont., 20(1), 141 - 146. Obtenido de http://journal.upao.edu.pe/PuebloContinente/article/viewFile/562/524

Ríos, R. (2017). Metodología para la Investigación y Redacción (Primera ed.). Malaga: Servicios Académicos Intercontinentales S.L.

Romero, R., Barboza, L., Cueva, H., Arenas, A., \& Cuevas, H. (2016). Estrategia instruccional basada en el método Pilve para la comprensión lectora de los estudiantes universitarios. Impacto Científico, 11(1), 37 -52. Recuperado el 15 de 03 de 2018

Ruíz, B. (2008). Lectura Efectiva. Trabajo de Ascenso. Lectura Efectiva., Universidad Rafael Landivar. Guatemala.

Universia Argentina. (06 de Julio de 2016). www.universia.com.ar. Recuperado el 15 de 03 de 2018 , de www.universia.com.ar: http://noticias.universia.com.ar/educacion/noticia/2016/07/06/1141486/lectura-rapidaaprende-leer-manera-eficiente.html

Zorrilla, J. (2008). Evaluación de la comprensión lectora: dificultades y limitaciones. Instituto Nacional de Evaluación y Calidad del Sistema Educativo, Caracas - Venezuela. 